\title{
Detección de Anticuerpos contra Ehrlichia spp en Propietarios de Caninos Domésticos con Ehrlichiosis
}

\author{
Detection of Antibodies Against Ehrlichia spp in Owners of Domestic Dogs with \\ EHRLICHIOSIS
}

\author{
Beatriz Gómez M. ${ }^{1}$, Olga Li E. ${ }^{1,4}$, Luis Hoyos S. ${ }^{1}$, Alberto Manchego S. ${ }^{2}$, \\ Francisco Suárez A. ${ }^{3}$
}

\section{Resumen}

Se determinó la seropositividad contra Ehrlichia chaffeensis en propietarios de perros con antecedentes de ehrlichiosis mediante la prueba de inmunofluorescencia indirecta (IFI) y su asociación con el género, edad, exposición a garrapatas y nivel de contacto con los perros. El estudio se realizó entre enero y diciembre de 2009, donde se evaluaron 95 personas de Lima Metropolitana (Perú), sin distinción de género, edad o condición socioeconómica y cuyos perros tenían historia de ehrlichiosis menor de un año. El $31.6 \%$ (30/95) presentaron anticuerpos contra E. chaffeensis. El 38.7\% (24/62) de los individuos menores a 40 años y el 18.2\% (6/33) de los individuos mayores a 40 años resultó ser seropositivo a $E$. chaffeensis $(\mathrm{p}<0.05)$. El $29.4 \%$ de varones y el $32.7 \%$ de mujeres resultaron seropositivos a E. chaffeensis ( $\mathrm{p}<0.05)$. Además, el 40.0 y $8.0 \%$ de las personas expuestas y no expuestas a artrópodos presentaron seropositividad a este patógeno.

Palabras clave: Ehrlichia chaffeensis; inmunofluorescencia indirecta; personas; garrapatas; perros

\section{AbstraCt}

The seroreaction of Ehrlichia chaffeensis through the indirect immunofluorescence test (IFI) was determined in owners of dogs with a history of ehrlichiosis. In addition, the association with gender, age, exposure to ticks and level of contact with dogs was evaluated. The study was conducted between January and December 2009, where 95 people from Lima Metropolitan Region (Peru) were evaluated, regardless of gender, age

\footnotetext{
${ }^{1}$ Laboratorio de Patología Clínica y Biología Molecular, ${ }^{2}$ Laboratorio de Microbiología y Parasitología Veterinaria, ${ }^{3}$ Laboratorio de Medicina Veterinaria Preventiva, Facultad de Medicina Veterinaria, Universidad Nacional Mayor de San Marcos, Lima, Perú

${ }^{4}$ E-mail: olgalie@hotmail.com
}

Recibido: 14 de noviembre de 2016

Aceptado para publicación: 28 de agosto de 2017 
or socioeconomic status, and whose dogs had a history of ehrlichiosis of less than one year. The results showed that $31.6 \%(30 / 95)$ of dog owners presented antibodies against E. chaffeensis. Besides, $38.7 \%$ (24/62) of the individuals younger than 40 years and $18.2 \%$ $(6 / 33)$ of individuals older than 40 years were seropositive to E. chaffeensis $(\mathrm{p}<0.05)$, $29.4 \%$ of males and $32.7 \%$ of females were seropositive to E. chaffeensis ( $<<0.05)$, and 40.0 and $8.0 \%$ of those exposed and not exposed to arthropods presented seropositivity to this pathogen.

Key words: Ehrlichia chaffeensis; indirect immunofluorescence; people; ticks; dogs

\section{INTRODUCCIÓN}

La ehrlichiosis es una enfermedad infecciosa producida por bacterias Gram negativas intracelulares obligadas de tipo pleomórficas y con frecuencia esferoide $\mathrm{u}$ ovoide (López et al., 2003). Estos gérmenes pertenecen al Orden Rickettsiales, Familia Anaplasmataceae y géneros Ehrlichia y Anaplasma (Dumler et al., 2001), los cuales infectan leucocitos y plaquetas (Dumler et al., 1993; Tamí, 2003). Entre ellas, existen seis especies que causan infecciones en humanos, incluyendo Ehrlichia chaffeensis, agente responsable de la Ehrlichiosis Monocítica Humana (EMH) y Anaplasma phagocytophilum, causante de la Anaplasmosis Granulocítica Humana (AGH). Así mismo, se han detectado pacientes infectados con E. ewingii, E. canis, E. equi y Neorickettsia sennetsu (Chen et al., 1994; Dumler et al., 2007).

La ehrlichiosis es una zoonosis emergente transmitida a los humanos a través de la picadura de garrapatas infectadas (Dumler y Bakken, 1995; Bojersson, 2000). La enfermedad es de gran importancia en países tropicales y sub-tropicales (Green, 1997). La primera infección por ehrlichias en humanos fue reconocida como «Fiebre del Sennetsu» y se describió en Japón en 1954. No obstante, el estudio de las ehrlichiosis humanas sufrió un espectacular avance en 1986 debido a la descripción del primer caso de EMH en los EEUU, en un paciente con sintomatología compatible con Fiebre Manchada de las Mon- tañas Rocosas (Maeda et al., 1987; Anderson et al., 1991).

En Chile y Argentina se identificaron anticuerpos contra Ehrlichia chaffeensis por primera vez en humanos en 1999 (López et al., 1999; Ripoll et al., 1999), encontrándose prevalencias de 11.8 y $14 \%$, respectivamente. Además, ha sido reportado en muestras serológicas de humanos en Venezuela (Pérez et al., 2006) y Brasil (Costa et al., 2006). La mayor incidencia de estas infecciones se produce en los meses de primavera-verano y principios de otoño, donde las especies de garrapatas implicadas en la transmisión de estas bacterias están más activas (Blanco y Oteo, 2002).

Moro et al. (2009) encontraron seroprevalencias para E. chaffeensis del 25, 23,3 y $3 \%$ en los departamentos de Piura, Cuzco, Lima e Iquitos, respectivamente; mientras que el Instituto Nacional de Salud (INS) reportó una seroprevalencia de $E$. chaffeensis de $9.2 \%$ en el departamento de Ancash (Anaya et al., 2009). En fechas más recientes, Paulino et al. (2013) identificaron en Lima Metropolitana anticuerpos contra $E$. chaffeensis $(20 \%)$ y E. canis $(23.3 \%)$ mediante serología (inmunofluorescencia indirecta) en personas que realizan actividades veterinarias (médicos veterinarios, técnicos de laboratorio, bañadores de perros, etc.). Por lo tanto, se cree que la exposición a ehrlichiosis puede representar un riesgo para los propietarios de mascotas que padecen o padecieron la enfermedad, lo cual podría tener gran repercusión en la salud pública. 
El presente estudio tuvo por objetivo determinar la seropositividad contra $E$. chaffeensis en propietarios de perros con ehrlichiosis y evaluar la asociación entre las variables sexo, edad, procedencia y exposición a garrapatas frente a la seropositividad contra ehrlichiosis.

\section{Materiales Y Métodos}

\section{Lugar del Estudio}

El estudio se realizó entre enero y diciembre de 2009 en la Facultad de Medicina Veterinaria (FMV) de la Universidad Nacional Mayor de San Marcos (UNMSM), Lima, Perú. La toma de muestras de pacientes y procesamiento de muestras se realizó en el Laboratorio de Patología Clínica Veterinaria, mientras que el ensayo de inmunofluorescencia indirecta (IFI) para el diagnóstico de E. chaffeensis se realizó en el Laboratorio de Virología.

\section{Población de Estudio}

La población del estudio estuvo conformada por 95 personas (propietarios de canes), los cuales ingresaron al estudio voluntariamente, firmando un consentimiento informado que detallaba los procedimientos a realizar y los objetivos del estudio. Los propietarios fueron seleccionados a partir de la llegada de perros con diagnóstico definitivo de ehrlichiosis canina a la Clínica de Animales Menores de la Facultad de Medicina Veterinaria de la UNMSM.

El diagnóstico definitivo del cuadro de ehrlichiosis en los perros fue realizado mediante evaluación clínica, resultados hematológicos y detección de anticuerpos contra Ehrlichia canis / Ehrlichia ewingii. Por cada perro se incluyó un máximo de 2 personas (propietarios) que manifestaban vivir permanentemente con el animal (adultos, adolescentes o niños). Los perros debían tener diagnóstico reciente, estar en pleno tra- tamiento contra ehrlichiosis o haber padecido la infección con un año de antigüedad como máximo.

Todos los procedimientos realizados en el presente estudio fueron previamente revisados y aprobados por el Comité de Ética de la FMV-UNMSM. El nombre de los propietarios se mantuvo anónimo para los efectos del estudio.

\section{Datos y Muestras}

En un formulario, se colectaron datos de los participantes con relación a la edad, género, procedencia, antecedentes de mordedura de perros y contacto con garrapatas. Los formularios fueron llenados y firmados por los participantes, y en el caso de niños fue llenado por sus padres.

Se tomaron aproximadamente $6 \mathrm{ml}$ de sangre de los pacientes (personas) mediante sistema de sangrado al vacío en tubos sin anticoagulante. Las muestras fueron incubadas en baño maría a $37^{\circ} \mathrm{C}$ durante $20 \mathrm{~min}$ para favorecer la formación del coágulo sanguíneo. Luego, se centrifugaron a 1600 g por $10 \mathrm{~min}$. El suero resultante fue colectado en viales de $2 \mathrm{ml}$ y almacenado en congelación $\left(-20^{\circ} \mathrm{C}\right)$ hasta la realización de las pruebas serológicas.

\section{Inmunofluorescencia Indirecta (IFI)}

Se realizó la prueba de inmunofluorescencia indirecta (IFI). Se utilizaron placas conteniendo células infectadas (DH82) con Ehrlichia chaffeensis (Fuller Laboratories $\left.{ }^{\circledR}\right)$. Se utilizó una anti-inmunoglobulina (anti-Ig-FITC) conjugada con el marcador fluorescente para la lectura final (Paulino et al., 2013; Starkey et al., 2014). El punto de corte para la prueba fue de 1:50. La lectura de las placas se hizo en un microscopio de epifluorescencia con filtro azul Leica, empleando el objetivo de 40X y 100X (Figura 1A). Se adjudicó una escala cualitativa (de una a cuatro cruces) para las muestras posi- 


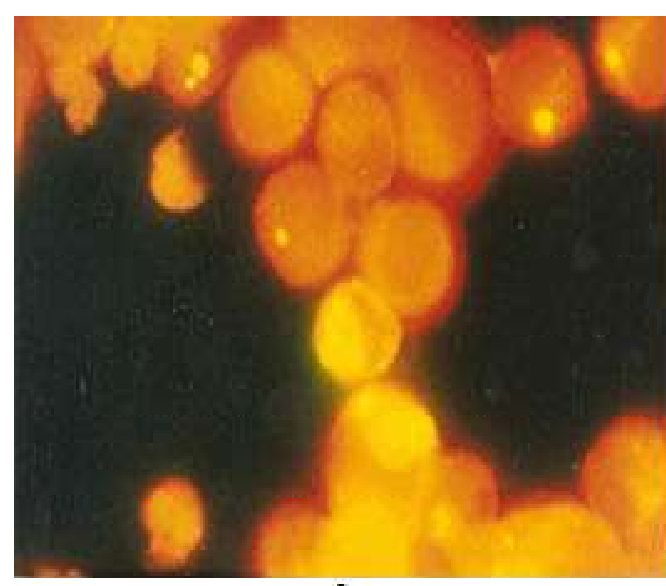

A

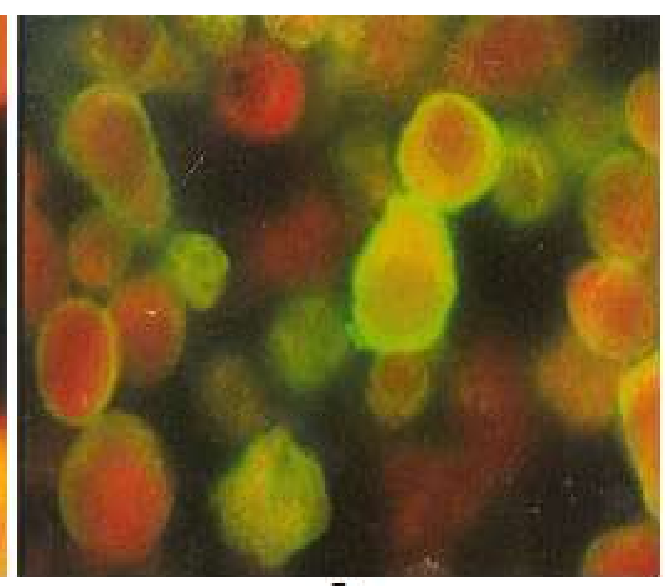

B

Figura 1. Prueba de inmunofluorescencia indirecta (IFI) contra Ehrlichia chaffeensis (Dilución 1:50). A) Resultado negativo; B) Resultado positivo. 400X

tivas comparándolas con el control positivo. Asimismo, se consideraron sueros negativos a aquellos que no presentaron fluorescencia alguna, observándose únicamente el color rojizo ocre de las células (color de contraste), al igual que en la imagen del control negativo (Figura 1B).

\section{Análisis Estadístico}

Se calculó el porcentaje de positividad a E. chaffeensis con intervalos de confianza de $95 \%$. Asimismo, se determinaron los porcentajes de positividad de acuerdo con las variables edad (mayores o menores de 40 años), género, procedencia y exposición a garrapatas (Sí, No) en un cuadro de contingencia.

Se utilizó la prueba de Chi-cuadrado para evaluar la asociación estadística entre las variables antes descritas y el porcentaje de positividad a $E$. chaffeensis, considerando un nivel de significancia de 5\%. Todos los análisis fueron realizados con el paquete estadístico Stata 14 (Stata Corp, College Station, TX).

\section{Informes a los Pacientes}

Los resultados serológicos fueron enviados a los participantes del estudio utilizan- do los correos electrónicos personales registrados en las fichas correspondientes.

\section{Resultados}

Treinta de los 95 propietarios de mascotas resultaron positivos a la presencia de anticuerpos contra $E$. chaffeensis, lo que representó una prevalencia de $31.6 \%$ (IC95\%: 22.4-41.9\%).

El 38.7\% (24/62) de los individuos menores a 40 años resultó ser seropositivo, mientras que el $18.2 \%(6 / 33)$ de los individuos mayores a 40 años presentó seropositividad a E. chaffeensis, según la técnica de IFI $(\mathrm{p}<0.05)$. Asimismo, el 29.4\% de varones y el $32.7 \%$ de mujeres resultaron seropositivos a E. chaffeensis $(\mathrm{p}<0.05)$. Además, el 40.0 y $8.0 \%$ de las personas expuestas y no expuestas a artrópodos presentaron seropositividad a este patógeno (Cuadro 1).

Respecto a la evaluación de la asociación estadística de la edad, género y exposición a garrapatas frente a la seropositividad, se encontró que las edades menores a 40 años y la exposición a artrópodos (garrapatas) presentó tener asociación estadística frente a la 
Cuadro 1. Resultados de la prueba de Inmunofluorescencia Indirecta (IFI) a Ehrlichia chaffeensis en 95 propietarios de perros con historia de ehrlichiosis ( $<1$ año)

\begin{tabular}{lcc}
\hline & \multicolumn{2}{c}{ Pacientes } \\
\cline { 2 - 3 } Variables & Total & $\begin{array}{c}\text { Positivos } \\
(\%)\end{array}$ \\
\hline Edad (años) & & \\
$\quad \leq 40$ & 62 & $38.7^{\mathrm{a}}$ \\
$\quad>40$ & 33 & $18.2^{\mathrm{b}}$ \\
Género & & \\
$\quad$ Masculino & 34 & $29.4^{\mathrm{a}}$ \\
$\quad$ Femenino & 31 & $32.7^{\mathrm{b}}$ \\
Exposición a & & \\
garrapatas & & \\
$\quad$ Sí & 70 & $40.0^{\mathrm{a}}$ \\
$\quad$ No & 25 & $8.0^{\mathrm{b}}$ \\
\hline Total & 95 & 100.0 \\
\hline a,b Superíndices & diferentes & dentro de \\
variables indican & diferencia & estadística \\
(p<0.05) & &
\end{tabular}

seropositividad contra Ehrlichia chaffeensis en el presente estudio.

\section{Discusión}

Los resultados del estudio indicaron un elevado porcentaje de positividad a ehrlichiosis en propietarios de canes con antecedentes de enfermedad (31.6\%), valores mayores al 9.2\% reportado por Anaya et al. (2009) en Ancash y las seroprevalencias de 3 a $25 \%$ reportadas por Moro et al. (2009) en diversas zonas del Perú. No obstante, dichos estudios fueron realizados con población rural y no necesariamente con vínculos a perros con antecedentes de la enfermedad. El estudio más parecido al presente es el llevado a cabo por Paulino et al. (2013), quienes demostraron $20 \%$ de positividad a $E$. chaffeensis en una población ocupacional en riesgo, tales como médicos veterinarios, bañadores de perros, enfermeros veterinarios y técnicos en general.

En el presente estudio se halló que las personas menores a 40 años presentaron una mayor frecuencia de seropositividad contra E. chaffeensis que personas de mayor edad; sin embargo, otros estudios indican que las edades de mayor riesgo son superiores a 40 años, ya que esta enfermedad es considerada «ocupacional» (Paddock y Childs, 2003), reportándose incluso la edad de 53 años como edad promedio (Ismail et al., 2010). En el presente estudio se postula la idea que la seropositividad está vinculada al mayor contacto doméstico con animales infectados, lo que explicaría la mayor proporción en personas menores a los 40 años. El CDC (2010) indica en su página web que en el periodo 2000-2010, las personas entre 40 y 44 años presentaron una probabilística de 1.8 casos de ehrlichiosis por millón de habitantes, mientras que las personas entre 60 y 64 años tuvieron una probabilística de 4.7 casos por millón.

El estudio reveló una mayor proporción de mujeres seropositivas a $E$. chaffeensis. Sin embargo, Paddock y Childs (2003) indican una ratio de 2:1 a favor de los varones. Inclusive el CDC (2010) reporta esta tendencia debido a la asociación entre la ehrlichiosis y las actividades laborales/recreacionales que realizan los hombres en zonas prevalentes (Ismail et al., 2010), llegando al 61\% de frecuencia para los varones.

El factor de riesgo de mayor impacto es la exposición a garrapatas debido a que son los transmisores de la enfermedad. Esto ha sido ampliamente corroborado con estudios relacionados (Anaya et al., 2009; Moro et al., 2009; Paulino et al., 2013). Los géneros que principalmente están involucrados en la transmisión de la ehrlichiosis son Riphicephalus sp, Amblyomma sp e Ixodes $\mathrm{sp}$, los cuales han sido reportados en la costa peruana (Bustamante, 1998; Liberato, 1998; Estares, 1999). 


\section{Conclusiones}

- $\quad$ Se reporta una seropositividad a $E$. chaffeensis de 31.6\% ( $\left.\mathrm{IC}_{95 \%}: 22.4-41.9\right)$ en propietarios de canes con antecedentes de ehrlichiosis en Lima Metropolitana.

- Las personas menores a 40 años presentaron una mayor seropositividad contra Ehrlichia chaffeensi que personas de mayor edad.

\section{Literatura Citada}

1. Anaya E, Morón C, Jaramillo K, Mendoza L, Román R. 2009. Evidencia serológica de Ehrlichiosis humana en Ancash, Perú. Rev Peru Med Exp Salud Publica 26: 54-57.

2. Anderson B, Dawson J, Jones D, Wilson K. 1991. Ehrlichia chaffeensis, a new species associated with human ehrlichiosis. J Clin Microbiol 29: 28382842.

3. Blanco J, Oteo J. 2002. Human granulocytic ehrlichiosis in Europe. Clin Microbiol Infect 8: 763-672. doi: 10.1016/ S1433-1128(04)80006-8

4. Breitschwerdt E, Hegarty B, Hancock S. 1998. Sequential evaluation of dogs naturally infected with Ehrlichia canis, Ehrlichia chaffeensis, Ehrlichia equi, Ehrlichia ewingii, or Bartonella vinsonii. J Clin Microbiol 36: 2645-2651.

5. Bojersson D. 2000. Ehrlichiosis: new perspectives on pathogenesis and diagnosis. Proc $18^{\text {th }}$ American College of Veterinary Internal Medicine. Seattle, USA.

6. Bustamante A. 1998. Prevalencia de ectoparásitos en Canis familiaris en la zona climática litoral de Lima Metropolitana en la estación de invierno. Tesis de Médico Veterinario. Lima. Univ Nacional Mayor de San Marcos. 43 p.

7. [CDC] Center for Disease Control and Prevention. 2010. Human ehrlichiosis and anaplasmosis. Available in : http:// www.cdc.gov/anaplasmosis/, http:// www.cdc.gov/ehrlichiosis/

8. Chen S, Dumler J, Bakken J, Walker D. 1994. Identification of a granulocytotropic Ehrlichia species as the etiologic agent of human disease. J Clin Microbiol 32: 589-595.

9. Costa P, Valle L, Brigatte M, Greco D. 2006. More about human monocytotropic ehrlichiosis in Brazil: serological evidence of nine new cases. Brazil J Infect Dis 10: 7-10. doi: 10.1590/S141386702006000100002

10. Costa P. 2004. Serologic evidences of Rickettsia rickettsii, Rickettsia typhi, Coxiella burnetii, Bartonella quintana, Bartonella henselae and Ehrlichia chaffeensis infections in healthy individuals and febrile aids and non-AIDS patients from the region of Juiz de Fora, Minas Gerais. Rev Inst Med Trop Sao Paulo 47: 208. doi: 10.1590/S003646652005000400015

11. Dumler J, Madigan J, Pusterla N, Bakken J. 2007. Ehrlichiosis in humans: epidemiology, clinical presentation, diagnosis, and treatment. Clin Infect Dis 45(Suppl 1): S45-S51. doi: 10.1086/ 518146

12. Dumler J, Barbet A, Bekker C, Dasch G, Palmer G, Ray S, et al. 2001. Reorganization of genera in the families Rickettsiaceae and Anaplasmataceae in the order Rickettsiales: unification of some species of Ehrlichia with Anaplasma, Cowdria with Ehrlichia and Ehrlichia with Neorickettsia, descriptions of six new species combinations and designation of Ehrlichia equi and 'HGE agent' as subjective synonyms of Ehrlichia phagocytophila. Int $\mathrm{J}$ Sys Evol Microbiol 51:2145-2165. doi: 10.1099/ 00207713-51-6-2145

13. Dumler J, Bakken J. 1995. Ehrlichial diseases of humans: emerging thick-borne infections. Clin Infect Dis 20: 11021110. doi: $10.1093 /$ clinids/20.5.1102 
14. Dumler J, Dawson J, Walker D. 1993. Human ehrlichiosis: hematopathology and immunohistologic detection of Ehrlichia chaffeensis. Hum Pathol 24: 391-396. doi: 10.1016/0046-8177(93) 90087-W

15. Estares L. 1999. Prevalencia de ectoparásitos de Canis familiaris en los distritos de San Juan de Lurigancho, San Martín de Porres, Comas e Independencia de Lima Metropolitana. Lima. Tesis de Médico Veterinario. Univ Nacional Mayor de San Marcos. $61 \mathrm{p}$.

16. Frank J, Breitschwerdt E. 1999. A retrospective study of ehrlichiosis in 62 dogs from North Carolina and Virginia. J Vet Intern Med 13: 194-201.

17. Green R. 1997. Ehrlichiosis canina: implicancias clínicas de factores humorales. En Kirk K (ed). Terapéutica veterinaria de pequeños animales. $12^{\mathrm{a}} \mathrm{ed}$. México: McGraw-Hill Interamericana. $p$ 317-320.

18. Harkess J. 1989. Ehrlichiosis: a cause of bone marrow hypoplasia in humans. Am J Hematol 30: 265-267.

19. Ismail N, Bloch K, McBride J. 2010. Human ehrlichiosis and anaplasmosis. Clin Lab Med 30: 261-292. doi: 10.1016/ j.cll.2009.10.004

20. Liberato $W$. 1998. Prevalencia de ectoparásitos en Canis familiaris en los distritos de San Juan de Miraflores, Villa María del Triunfo y Villa el Salvador. Lima. Lima. Tesis de Médico Veterinario. Univ Nacional Mayor de San Marcos. $31 \mathrm{p}$.

21. López D, Rivera S, Concha G, Gatica Q, Loeffeholz M, Barriga V. 2003. Ehrlichiosis humana en Chile: evidencia serológica. Rev Med Chile 131: 67-70. doi: 10.4067/S0034-98872003000100010

22. López J, Castillo A, Muñoz M, Hildebrandt S. 1999. Hallazgo de Ehrlichia canis en Chile, informe preliminar. Arch Med Vet 31: 211-214. doi: 10.4067/S0301-732X1999000200008

23. Maeda K, Markowitz N, Hawley RC, Ristic M, Cox D, McDade JE. 1987. Human infection with Ehrlichia canis, a leukocytic rickettsia. N Engl J Med 316:
853-856. doi: 10.1056/NEJM19870 4023161406

24. Moro P, Shah J, Li O, Gilman R, Harris N, Moro M. 2009. Short report: serologic evidence of human ehrlichiosis in Peru. Am J Trop Med Hyg 80: 242244.

25. Ndip L, Ndip R, Esemu S, Walker D, McBride J. 2010. Predominance of Ehrlichia chaffeensis in Rhipicephalus sanguineus ticks from kennel-confined dogs in Limbe, Cameroon. Exp App Acarol 50: 163-168. doi: 10.1007/s10493009-9293-8

26. Paddock C, Childs J. 2003. Ehrlichia chaffeensis: a prototypical emerging pathogen. Clin Microbiol Rev 16: 37-64. doi: 10.1128/CMR.16.1.37-64.2003

27. Paulino A, Li O, Hoyos L, Suárez F, Díaz D. 2013. Detección serológica de Ehrlichia canis y Ehrlichia chaffeensis en personal de clínicas veterinarias en Lima Metropolitana. Rev Inv Vet Peru 24: 217-221. doi: 10.15381/ rivep.v24i2.2505

28. Perez M, Bodor M, Zhang C, Xiong Q, Rikihisa Y. 2006. Human infection with Ehrlichia canis accompanied by clinical signs in Venezuela. Ann N Y Acad Sci 1078: 110-107. doi: 10.1196/ annals. 1374.016

29. Rikihisa Y. 1999. Clinical and biological aspects of infection caused by Ehrlichia chaffeensis. Microb Infect 1: 367-376.

30. Ripoll C, Remondegui C, Ordonez G, Arazamendi R, Fusaro H, Hyman MJ, et al. 1999. Evidence of rickettsial spotted fever and ehrlichial infections in a subtropical territory of Jujuy, Argentina. Am J Trop Med Hyg 61: 350-354.

31. Rohrbach B, Harkess J, Ewing S, Kudlac J, McKee G, Istre G. 1990. Epidemiologic and clinical characteristics of persons with serologic evidence of $E$. canis infection. Am J Public Health 80: 442-445.

32. Stone JH, Dierberg K, Aram G, Dumler J. 2004. Human monocytic ehrlichiosis. JAMA 292: 2263-2270. doi: 10.1001/jama.292.18.2263 
33. Tamí I. 2003. Ehrlichiosis humana: Ehrlichia trombocítica en sangre periférica. Rev Soc Ven Microbiol 23: 135-141.
34. Zhang $X$, Zhang J, Long S, Ruble R, $Y u X$. 2003. Experimental Ehrlichia chaffeensis infection in beagles. J Med Microbiol 52: 1021-1026. doi: 10.1099/ jmm.0.05234-0 